Research Article

\title{
Acceleration Response First Passage Failure Probability Analysis Method for Nonlinear Package
}

\author{
Dapeng Zhu \\ School of Traffic and Transportation, Lanzhou Jiaotong University, Lanzhou 730070, China \\ Correspondence should be addressed to Dapeng Zhu; zhudapeng@mail.lzjtu.cn
}

Received 8 May 2018; Accepted 4 July 2018; Published 30 July 2018

Academic Editor: Tai Thai

Copyright ( 2018 Dapeng Zhu. This is an open access article distributed under the Creative Commons Attribution License, which permits unrestricted use, distribution, and reproduction in any medium, provided the original work is properly cited.

\begin{abstract}
The acceleration response first passage failure problem of the nonlinear package base excited by Gaussian white noise is analyzed. The model correction factor method (MCFM) is implemented in conjunction with the first-order reliability method (FORM) to analyze the first passage failure probability of the nonlinear package. The white noise is discretized in standard normal space, and an iterative algorithm is proposed to find the design point of the packaging system. On the design point, the hypersurface representing the limit-state function of the nonlinear package is replaced approximately by a hyperplane representing the limitstate function of an equivalent linear system, and the FORM is employed to calculate the failure probability of the packaging system. The accuracy of this method is verified by crude Monte Carlo simulations. Numerical simulations are carried out to observe the effects of system parameters variations on failure probability which can be used for the improvement of packaging design.
\end{abstract}

\section{Introduction}

In the process of product distribution from manufacturers to customers, protective package is designed to withstand the real operational conditions to which the product will be subjected. The main operational conditions include drop shock, random vibration from trucks, ships, or airplanes, and static compression. It is important and necessary to analyze the response of the product in the package under various operational conditions, based on the response analysis, and a proper packaging design may be carried out to increase package safety and reliability and avoid over-package costs. In the package distribution process, if the package is excited by drop shock, the motion state of the package changes greatly in relatively short time; therefore, in the drop shock excitation conditions, the package is more inclined to get failed.

In recent years, many researchers have devoted their attentions to the package safety and cushion material dynamic properties under drop impact condition. Wang and Jiang $[1,2]$ used the two-degree-of-freedom mechanical system to represent the packaging system; the response of the key component in the product was analyzed numerically, the dropping damage boundary curve was developed, and the effects of system parameters variations on the damage boundary curve were also observed. Zhong et al. [3] took the friction force into account and proposed a new friction equivalent drop theory; this theory provided a new theoretical basis for experimental analysis of structural cushioning materials. To model the closed-cell foam dynamic properties efficiently in the finite element method, Piatkowski and Osowski [4] presented a modified method for the nonlinear dynamic stress-strain curve determination with the use of cushion curves. In $[5,6]$, new methods for evaluating cushioning properties in drop shock conditions were also presented. Lu and Gao [7] proposed the visual mass method and analyzed quasi-static compression and drop impact behaviors of the multilayered corrugated paperboard cushioning structure. In order to evaluate safety of the package excited by shock, the nonlinear packaging system was modeled as a lumped mass system, the shock responses of the key component were investigated numerically, and the effects of system parameters variation were also discussed in [8-10].

In the package distribution process, most of the time, it is the random vibration that excites the packaging system; if the 
acceleration response of the package reaches or exceeds a certain fixed threshold, that is, the fragility of the product in the package, package failure will occur. This package failure mode is the first passage failure, also mentioned as the first excursion failure, which is a major failure mode of a structure. The main goal of this paper is to analyze the first passage failure probability under random excitation conditions, and the influence of system parameters on the failure probability was also discussed. Based on the analytical conclusions, the package can be designed properly to prevent failure or to minimize the likelihood of the first passage failure occurrence. For the nonlinear package excited by random vibration, nonlinear stochastic dynamic analysis methods can be employed to estimate failure probability. A variety of methods for nonlinear stochastic dynamic analysis have been explored and presented. In the structural reliability field, first- and second-order reliability methods (FORM and SORM) [11, 12] have been proven to be effective methods, for their simplicity and efficiency. The mirror image excitation method presented by Koo et al. [13] provided an approximate method for the nonlinear system failure probability estimation, and the solution of this method does not require the finding of the design point and response gradient; however, this method is restricted to the displacement first passage of single-degreeof-freedom problems. In the tail equivalent linearization method (TELM) $[14,15]$, the nonlinear system was equivalently replaced by a linear system at the design point; this method can be adopted in many nonlinear cases with great accuracy, especially for high response threshold cases. In recent years, a promising method for the structural reliability problems is the response surface methodology [16, 17]; in this method, the discrete points on the hypersurface around the design point were discretized and obtained, and the points were used to reconstruct a surface to replace the actual hypersurface relating to the real system.

The most important step in the nonlinear stochastic dynamic analysis is the determination of the design point. In this paper, a correction idealized system is developed to equivalently replace the real system, and an iterative algorithm is proposed for determining the design point of the nonlinear packaging system, based on the model correction factor method (MCFM). A hyperplane representing the limit-state function of a linear system is used to replace the hypersurface representing the limit-state function of the actual nonlinear system. The FORM solution of the packaging system is used to estimate the reliability approximately. Through the comparison analysis between the crude Monte Carlo simulation and the results from MCFMFORM, it is shown the presented MCFM-FORM approach allows accurate first passage failure probability estimation. Finally, the proposed MCFM-FORM is applied to analyze the effects of parameters variations on nonlinear package reliability.

\section{Acceleration Response First Passage of the Packaging System}

The package is modeled by a single-degree-of-freedom system, which in shown in Figure 1. The rigid mass in the model represents the product in the package, and the spring, paralleled with a damping, represents the cushion material. In the process of package distribution, the package is excited by the base random acceleration vibration input $\ddot{b}$. In order to apply structural reliability methods to the package reliability problems, it is necessary to discretize the random input process and represent it in terms of a finite number of random variables. For the zero-mean base input, the acceleration excitation $\ddot{b}$ can be represented as

$$
\ddot{b}(t)=\sum_{i=1}^{n} s_{i}(t) u_{i}=\mathbf{s}(t) \cdot \mathbf{u},
$$

where $\mathbf{u}=\left[u_{1}, u_{2}, \ldots, u_{n}\right]^{T}$ is an $n$-dimensional standard normal vector, $\mathbf{s}(t)=\left[s_{1}(t), s_{2}(t), \ldots, s_{n}(t)\right]^{T}$ is a row vector of deterministic basis functions dependent on the covariance structure of the input process, $n$ is a measure of the resolution of the representation, and $T$ denotes the matrix transposition. As presented by (1), the randomness of the acceleration input $\ddot{b}$ is determined by the vector $\mathbf{u}$, and the time-evolution property of $\ddot{b}$ is presented by the vector $\mathbf{s}$. At a given time constant $t$, the excitation $\vec{b}$ can also be represented by a specified point in the standard normal space $\mathbf{u}$; on the contrary, a given point in the space $\mathbf{u}$ denotes a random input.

From (1), the input of the packaging system is a function of the standard normal variable $\mathbf{u}$, and the acceleration response of the package can also be represented as a function of $\mathbf{u}$. In order to solve the package acceleration response first passage problem, the limit-state function of this problem should be formulated firstly. For the acceleration response first passage of the package failure event, that is, at a specified time $t$, the acceleration response of the product in the package exceeds a specified product fragility $G_{c}$, and the limit-state function for this problem can be presented by

$$
g\left(\mathbf{u}, G_{c}, t\right)=G_{c}-\ddot{x}(\mathbf{u}, t) .
$$

The limit-state function $g$ can also be presented in the standard normal space $\mathbf{u}$. For the nonlinear packaging system, $g$ can be represented by a hypersurface in the space u. Therefore, the space $\mathbf{u}$ is divided by the hypersurface representing the limit-state function $g$ into two parts: in the first part, $g>0$, the package is safe, and in the other part of the space, $g \leq 0$, the acceleration response first passage of the package failure event occurs and the product in the package gets failed. The limit-state function, the safety region, and the failure region in the $\mathbf{u}$ space are shown in Figure 2. For the case $g \leq 0$, in all the realizations of $\mathbf{u}$ that give rise to the event $\left\{\ddot{x}(t, \mathbf{u}) \geq G_{c}\right\}$, the one that has the highest likelihood is the one nearest to the origin (Figure 2). This point known as the design point in the theory of structural reliability is defined as

$$
\begin{aligned}
\mathbf{u}^{*} & =\min \{\|\mathbf{u}\|\}, \\
\text { s.t. } g\left(\mathbf{u}, G_{c}, t\right) & =G_{c}-\ddot{x}(\mathbf{u}, t)=0,
\end{aligned}
$$

where $\|\cdot\|$ denotes the norm of a vector. In the FORM, a hyperplane is used to replace the actual limit-state hypersurface at the design point; applying the FORM to the 


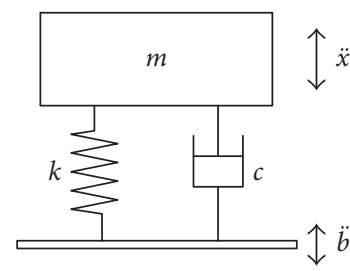

Figure 1: Simplified single-degree-of-freedom package model.

acceleration response first passage probability estimation, one obtains

$$
\operatorname{Pr}\left[\ddot{x}(\mathbf{u}, t) \geq G_{c}\right] \cong \Phi\left[-\beta\left(G_{c}, t\right)\right],
$$

where $\Phi[\cdot]$ denotes the standard normal cumulative probability function and $\beta\left(G_{c}, t\right)$ is the reliability index and is also the distance between the design point and origin; the reliability index can be presented as

$$
\begin{aligned}
\beta\left(G_{c}, t\right) & =\alpha\left(G_{c}, t\right) \cdot \mathbf{u}^{*}\left(G_{c}, t\right) \\
& =-\frac{\nabla_{\mathbf{u}} g\left(\mathbf{u}, G_{c}, t\right)}{\left\|\nabla_{\mathbf{u}} g\left(\mathbf{u}, G_{c}, t\right)\right\|} \cdot \mathbf{u}^{*}\left(G_{c}, t\right),
\end{aligned}
$$

where $\alpha\left(G_{c}, t\right)$ is the negative normalized gradient vector of the limit-state function at the design point (Figure 2).

In the first step, we assume the packaging system is linear, which means the stiffness coefficient $k$ and the damping coefficient $c$ are constant; therefore, the motion equation of the package can be given by

$$
m \ddot{z}+c \dot{z}+k z=m \ddot{b},
$$

where $z=b-x$ is the relative displacement of the product in the package. Applying the Duhamel integral, we can represent the acceleration response of the package as

$$
\begin{aligned}
\ddot{x}(t)= & 2 \xi \omega_{\mathrm{n}} \dot{z}+\omega_{\mathrm{n}}^{2} z=2 \xi \omega_{\mathrm{n}} \int_{0}^{t} \ddot{b}(\tau) h_{\dot{z}}(t-\tau) d \tau \\
& +\omega_{\mathrm{n}}^{2} \int_{0}^{t} \ddot{b}(\tau) h_{z}(t-\tau) d \tau=\int_{0}^{t} \ddot{b}(\tau) h(t-\tau) d \tau,
\end{aligned}
$$

where $\omega_{\mathrm{n}}$ and $\zeta$ are the natural frequency and damping ratio, respectively, and $h_{z}(t)$ and $h_{\dot{z}}(t)$ are the unit impulse displacement response and unit impulse velocity response functions; similarly, $h(t)$ is the unit impulse acceleration response function of the packaging system and can be given by

$$
\begin{aligned}
h(t)= & 2 \xi \omega_{\mathrm{n}} \exp \left(-\xi \omega_{\mathrm{n}} t\right) \cos \omega_{\mathrm{d}} t \\
& +\frac{1-2 \xi^{2}}{\sqrt{1-\xi^{2}}} \omega_{\mathrm{n}} \exp \left(-\xi \omega_{\mathrm{n}} t\right) \sin \omega_{\mathrm{d}} t,
\end{aligned}
$$

where $\omega_{\mathrm{d}}=\omega_{\mathrm{n}} \sqrt{1-\xi^{2}}$; from (1) and (7), one obtains

$$
\ddot{x}(t, \mathbf{u})=\int_{0}^{t} h(t-\tau) \sum_{i=1}^{n} s_{i}(\tau) u_{i} d \tau=\mathbf{a}(t) \cdot \mathbf{u},
$$

where $\mathbf{a}(t)=\left[a_{1}(t), a_{2}(t), \ldots, a_{n}(t)\right]^{T}$ is a vector, the elements of which are convolutions of the shape functions of

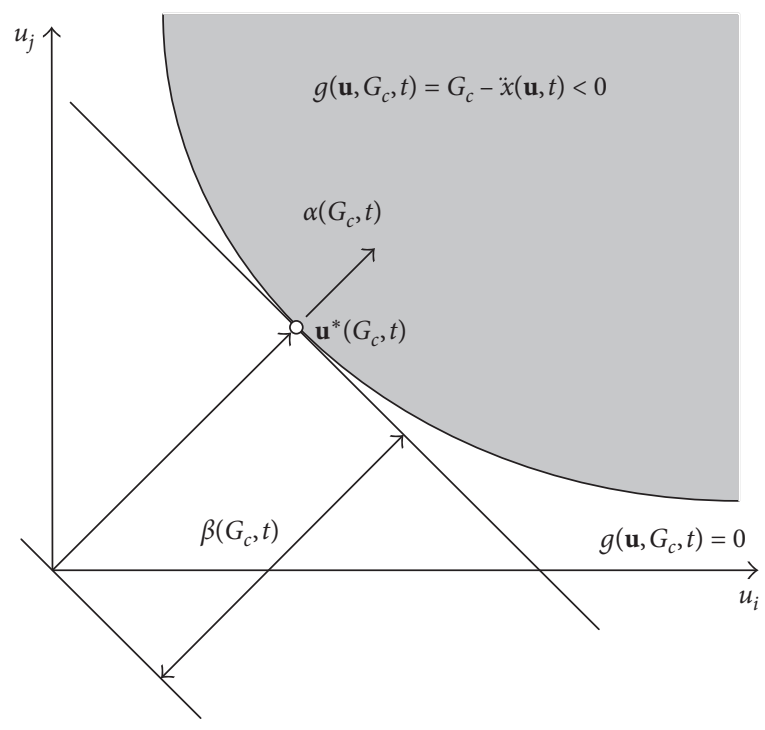

FIGURE 2: Vibration reliability analysis using FORM.

the excitation and the unit impulse acceleration response function of the system:

$$
a_{i}(t)=\int_{0}^{t} h(t-\tau) s_{i}(\tau) d \tau, \quad i=1,2, \ldots, n .
$$

From (9), one can obtain the limit-state function of the linear package base excited by random vibration by

$$
g\left(\mathbf{u}, G_{c}, t\right)=G_{c}-\mathbf{a}(t) \cdot \mathbf{u}=0 .
$$

From (11), the acceleration response of the linear packaging system is a linear function of standard normal random variables $\mathbf{u}$. Equation (11) defines the limit-state function surface of the linear package for the given fragility $G_{c}$ at time $t$, which defines a hyperplane in the space of $\mathbf{u}$; therefore, the design point can be expressed in the closed form as

$$
\mathbf{u}^{*}\left(G_{c}, t\right)=G_{c} \frac{\mathbf{a}(t)}{\|\mathbf{a}(t)\|^{2}} .
$$

And the solution for the reliability index is

$$
\beta\left(G_{c}, t\right)=\frac{G_{c}}{\|\mathbf{a}(t)\|} .
$$

Supposing we know the design point $\mathbf{u}^{*}\left(G_{c}, t\right)$ and solving for $\mathbf{a}(t)$ from (12), the gradient vector $\mathbf{a}(t)$ is obtained as

$$
\mathbf{a}(t)=G_{c} \frac{\mathbf{u}^{*}\left(G_{c}, t\right)}{\left\|\mathbf{u}^{*}\left(G_{c}, t\right)\right\|^{2}} .
$$

This expression indicates that, for a given vibration reliability problem, if the design point is obtained, one can determine the gradient vector $\mathbf{a}(t)$. On the contrary, using the components of $\mathbf{a}(t)$ in (10), it is possible to obtain a set of $n$ equations for determining the IRF of the linear system at a set of $n$ discrete time points.

From the discussion above, one can conclude that, for the linear packaging system excited by zero-mean vibration, 
its acceleration response can be determined by closed-form expression, its limit-state function is a linear function of standard normal random variables $\mathbf{u}$, and the limit-state function and reliability index can be expressed in the closed form. However, for the nonlinear packaging system, the acceleration response of the system cannot be obtained by steady closed-form expression; accordingly, the limit-state function and reliability index cannot be obtained in closedform expression either. An attractive and reasonable approach is to find an equivalent linear packaging system to replace the actual nonlinear system. This method $[18,19]$ can accurately estimate the second moments of the nonlinear packaging system response. However, the accuracy of this method in computing first passage probabilities is not good. Most commonly, the package response is assumed to have the Gaussian distribution, and a fairly good approximation of the response variance is obtained when the system nonlinearity is not severe. However, the accuracy deteriorates as the degree of system nonlinearity increases, particularly for the first passage probability, because the Gaussian assumption is not valid for such cases.

The discussion above highlights the importance of determining the design point of the nonlinear packaging system subjected to stochastic excitation. In this paper, a procedure based on the model correction factor method is formulated for determining the design point of the nonlinear packaging system, which can be adopted to analyze the first passage failure probability of the nonlinear packaging system.

\section{Model Correction Factor Method for Nonlinear System Dynamic Analysis}

The MCFM, originally formulated in [20], in nature, is a special form of the response surface method. Instead of selecting a more or less arbitrary mathematical form, in MCFM, the response surface is selected by an equivalent idealized system. The idealized system contains main features of the real nonlinear system. The hypersurface representing the limit-state function of the real system and hypersurface representing the limit-state function of the idealized system are brought to match at the neighbourhood of the design point in the $\mathbf{u}$ space. After some correction factor iterations, we can use the idealized system to replace the actual system approximately, and the idealized system can be used to analyze problems in vibration reliability. For the simplicity of the linear system in vibration reliability problems, we usually use a linear system as the idealized system.

Let $\ddot{x}_{\mathrm{r}}(\mathbf{u}, t)$ denote the acceleration response of the real system at the time constant $t$ to the base random vibration and $\ddot{x}_{\mathrm{i}}(\mathbf{u}, t)$ denote the acceleration response of the idealized system to the same excitation. Accordingly, the limit-state functions of the real and idealized systems $g_{\mathrm{r}}$ and $g_{\mathrm{i}}$ can be expressed:

$$
\begin{aligned}
g_{\mathrm{r}}\left[\ddot{x}_{\mathrm{r}}(\mathbf{u}, t), G_{c}\right] & =G_{c}-\ddot{x}_{\mathrm{r}}(\mathbf{u}, t)=0, \\
g_{\mathrm{i}}\left[\ddot{x}_{\mathrm{i}}(\mathbf{u}, t), G_{c}\right] & =G_{c}-\ddot{x}_{\mathrm{i}}(\mathbf{u}, t)=0 .
\end{aligned}
$$

The correction factor $v(\mathbf{u})$ is defined as the ratio of the acceleration response of the real system and idealized system:

$$
v(\mathbf{u})=\frac{\ddot{x}_{\mathrm{r}}(\mathbf{u}, t)}{\ddot{x}_{\mathrm{i}}(\mathbf{u}, t)} .
$$

Based on (16), we define a correction idealized system, whose limit-state function can be expressed as

$$
g_{\mathrm{i}}\left[\nu(\mathbf{u}) \ddot{x}_{\mathrm{i}}(\mathbf{u}, t), G_{c}\right]=G_{c}-v(\mathbf{u}) \ddot{x}_{\mathrm{i}}(\mathbf{u}, t)=0 .
$$

Substituting (16) into (17), we have

$$
g_{\mathrm{i}}\left[v(\mathbf{u}) \ddot{x}_{\mathrm{i}}(\mathbf{u}, t), G_{c}\right]=g_{\mathrm{r}}\left[\ddot{x}_{\mathrm{r}}(\mathbf{u}, t), G_{c}\right],
$$

which shows that the correction idealized system is completely identical to the real system. The correction idealized system is also difficult to solve in the direct way. However, because the two models describe the same system, the models behave in the same way; in this paper, by the reasonable approximate of the correction factor in the idealized model, we can obtain a good approximation for the real system.

At the design point $\mathbf{u}^{*}$ of the real system, the correction factor $\nu(\mathbf{u})$ can be expressed as the Taylor-series expansion, and we obtain

$$
\nu(\mathbf{u})=\nu\left(\mathbf{u}^{*}\right)+\nabla_{\mathbf{u}} \nu\left(\mathbf{u}^{*}\right)\left(\mathbf{u}-\mathbf{u}^{*}\right)+\cdots .
$$

By approximating the correction factor with the constant $v^{*}=v\left(\mathbf{u}^{*}\right)$ and substituting it in (17), the zero-order correction idealized model is obtained:

$$
g_{\mathrm{i}}\left[\nu^{*} \ddot{x}_{\mathrm{i}}(\mathbf{u}, t), G_{c}\right]=G_{c}-\nu^{*} \ddot{x}_{\mathrm{i}}(\mathbf{u}, t) .
$$

From (20), in order to find a good approximate of the idealized system, the task is then to reasonably find the value of the correction factor $v^{*}$ so that the estimated design point $u_{i}^{*}$ of the zero-order correction idealized model is as close as possible to the design point $\mathbf{u}^{*}$ of the real system. The estimation of the correction factor $v^{*}$ can be obtained by the following iterative algorithm:

Step 1. Select an idealized system. For the simplicity of the linear system in solving vibration reliability problems, in this paper, an equivalent linear system is selected as the idealized system, and the design point of this linear system is obtained by (12) and is taken as the initial iteration value $u_{i}^{(0)}$ of the real system design point. At the time constant $t$, the acceleration response of the idealized linear system is $G_{c}$, that is, $\ddot{x}_{i}^{(1)}=G_{c}$.

Step 2. Since each point in the standard normal space $\mathbf{u}$ can be considered as random excitation, the acceleration response of the real system $\ddot{x}_{\mathrm{r}}^{(1)}\left(u_{i}^{(0)}, t\right)$ at the time constant $t$ excited by the excitation corresponding to $u_{i}^{(0)}$ can be achieved by using numerical analysis.

Step 3. Using (16), calculate the initial estimate of the correction factor $v^{(1)}(\mathbf{u})=\ddot{x}_{\mathrm{r}}^{(1)} / \ddot{x}_{\mathrm{i}}^{(1)}$ and solve the corresponding correction idealized model $g_{\mathrm{i}}^{(1)}=$ $G_{c}-v^{(1)} \ddot{x}_{\mathrm{i}}(\mathbf{u}, t)$ by FORM to obtain its design point $u_{i}^{(1)}$ and the corresponding reliability index $\beta_{i}^{(1)}$ through use of (12) and (13), respectively. 
Step 4. Taking $u_{i}^{(1)}$ as the initial point of iteration, calculate the new correction factor $v^{(2)}: v^{(2)}(\mathbf{u})=$ $\ddot{x}_{\mathrm{r}}\left(u_{i}^{(1)}, t\right) / \ddot{x}_{\mathrm{i}}\left(u_{i}^{(1)}, t\right)$, and formulate the corresponding limit-state function of the new correction idealized model: $g_{\mathrm{i}}^{(2)}=G_{c}-v^{(2)} \ddot{x}_{\mathrm{i}}(\mathbf{u}, t)$; calculate the design point $u_{i}^{(2)}$ and the corresponding reliability index $\beta_{i}^{(2)}$ of this new model by FORM.

Step 5. Repeating Step 3 and Step 4, a sequence $\left(\nu^{(1)}, u_{i}^{(1)}\right),\left(\nu^{(2)}, u_{i}^{(2)}\right), \ldots$ is obtained. If the sequence converges, then $v^{(p)} \rightarrow 1$ and $\left\|u_{i}^{(p+1)}-u_{i}^{(p)}\right\|<\varepsilon$, where $\varepsilon$ is a set threshold. Then, $u_{i}^{(p)}$ can be considered as the design point of the correction idealized model. Furthermore, because $v^{(p)}=1$, we can conclude that $u_{i}^{(p)}$ is also located on the limit-state surface of the real system. If the acceleration response of the correction idealized model $\ddot{x}_{i}(\mathbf{u}, t)$ is not too far from the real model acceleration response, it is reasonable to expect the iteration of $u_{i}^{(p)}$ will lead to the convergence of the design point. On the contrary, if the sequence does not converge, then it suggests that the correction idealized model is too far from the real model. Therefore, it is necessary to select a reasonable correction idealized model.

From the steps above, one can obtain the design point of the nonlinear system by the MCFM iteration and FORM, and the above procedure normally converges if the correction idealized model is properly selected.

\section{Choice of the Correction Idealized Model and Optimization}

As described above, the most important step for the design point of the nonlinear system using MCFM is the proper selection of the correction idealized model. The linear system is an attractive selection, whose acceleration response, design point, and reliability index can all be obtained in the closed form, which have been discussed in the preceding part. The drawback of the linear idealized system is that as shown in Figure 3, the MCFM simply moves the limit-state hyperplane of the correction idealized model without changing its gradient. A linear system is selected as the correction idealized model; assume the design point of the system is $u_{i}^{(1)}$, the corresponding limit-state function of the model is $g_{i}^{(1)}$, which is indicated by the dashed line in Figure 3 , and the reliability index is $\beta_{i}^{(1)}$. In MCFM, the limit-state function of the correction idealized model can be obtained from (9) and (17):

$$
g_{i}\left[v^{*} \ddot{x}_{i}(\mathbf{u}, t), G_{c}\right]=G_{c}-v^{*} \mathbf{a}(t) \cdot \mathbf{u}=0 .
$$

From (21), we can observe that, for the linear correction idealized model, with the variation of $v^{*}$, the hyperplane $G_{c} / \nu^{*}-\mathbf{a}(t) \cdot \mathbf{u}=0$ moves in a direction parallel to $\mathbf{a}(\mathrm{t})$ until the design point $u_{i}^{*}=\left(G_{c} / \nu^{*}\right) \mathbf{a}(t) /\|\mathbf{a}(t)\|^{2}$ falls onto the limit-state hypersurface of the real model. It is obvious that the perfect estimation of the nonlinear system design point can be obtained only if the fixed vector $\mathbf{a}(\mathrm{t})$ is the same as the gradient vector of the real limit-state hypersurface at its design point. Since the design point $u_{i}^{*}$ is located on the

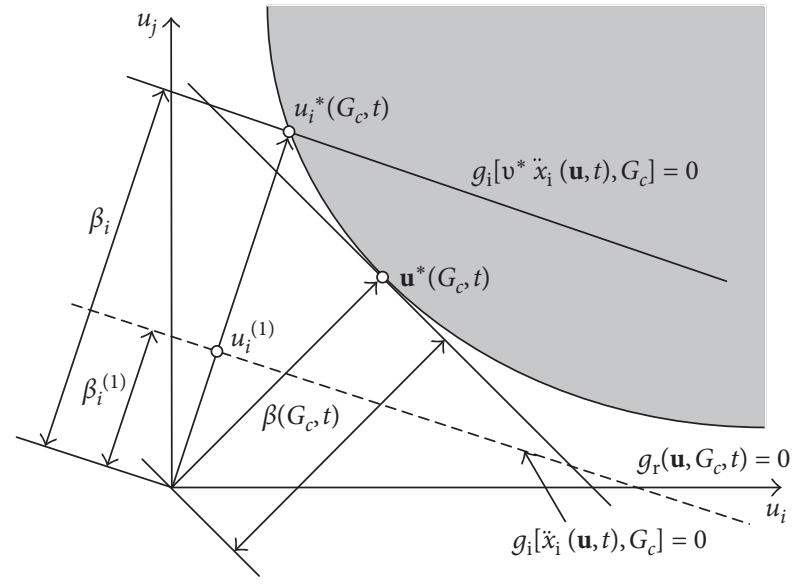

FIgURE 3: Design point determining using the linear correction idealized model.

limit-state surfaces of both the correction idealized and real models, the estimated reliability index $\beta_{i}$ is an upper bound to the reliability index of the real system; therefore, we obtain

$$
\beta \leq \beta_{i}
$$

where $\beta_{i}$ is the reliability index corresponding to the design point $\mathbf{u}_{i}^{*}$ in the iterations and $\beta$ is the reliability index corresponding to the real design point $\mathbf{u}^{*}$. From (22), one can conclude that $\|\beta\|=\min \left\{\left\|\beta_{i}\right\|\right\}$. Suppose we define the correction idealized linear system in terms of a set of $j$ parameters, $\boldsymbol{\theta}=\left[\theta_{1}, \theta_{2}, \ldots, \theta_{j}\right]$. For a given product fragility $G_{c}$, taking into account the significance of (22), we can obtain the optimal correction idealized linear system by solving the optimization problem:

$$
\boldsymbol{\theta}_{\text {opt }}\left(G_{c}\right)=\arg \min \left\{\beta_{i}(\boldsymbol{\theta})\right\},
$$

where $\beta_{i}(\boldsymbol{\theta})$ is the reliability index of the idealized linear system after achieving convergence according to the proposed algorithm. The optimization problem in (23) can be solved by the simplex method [20]. However, it is necessary to have a good starting point. For this purpose, we select a grid of potential starting points $\theta_{m}, m=1,2, \ldots$, compute the corresponding objective values $\beta_{i}(\boldsymbol{\theta})$, and select the starting point with the minimal $\beta$ value. From [20], we concluded that two points per parameter are sufficient. From the solution of (23), the optimized parameters $\boldsymbol{\theta}_{\text {opt }}$ for the correction idealized linear system can be obtained; the design point $\mathbf{u}^{*}$ corresponding to this optimized linear model is the design point of the real model.

\section{Example}

The package is modeled by a cubic nonlinear single-degreeof-freedom system, shown in Figure 1, and the motion equation of the system is

$$
m \ddot{z}+c \dot{z}+k z+k_{3} z^{3}=m \ddot{b},
$$

where $z$ is the compression of the cushion material, $z=b-x$; the basic parameters of the system are $m=10 \mathrm{~kg}$, $c=491 \mathrm{Ns} / \mathrm{m}, k=930100 \mathrm{~N} / \mathrm{m}$, and $k_{3}=3 \times 10^{10} \mathrm{~N} / \mathrm{m}^{3}$, and $\ddot{b}$ 
is the base acceleration excitation of the system. Assume $\ddot{b}$ is Gaussian white noise with the power spectral density $W(f)=0.1 \mathrm{~g}^{2} / \mathrm{Hz}$, where $g=9.8 \mathrm{~m} / \mathrm{s}^{2}$. Let $t_{i}=\Delta t \times i$, $i=0,1, \ldots, n$ denotes equally spaced discrete time points. The white noise can be approximately represented by a rectangular wave process defined by

$$
\ddot{b}(t) \cong \ddot{b} \ddot{b}_{i}=\frac{1}{\Delta t} \int_{t_{i-1}}^{t_{i}} \ddot{b}(\tau) d \tau, \quad t_{i-1}<t \leq t_{i},
$$

where $\Delta t$ is the time interval (in this paper, $\Delta t=0.001 \mathrm{~s}$ ) and $\ddot{b}_{i}$ denotes the intensity of the rectangular pulse in the time interval $t_{i-1}<t \leq t_{i}$. It is easy to show that $\ddot{b}_{i}$ are statistically independent normal random variables having a zero mean and the variance $\sigma=\sqrt{W(f) /(2 \Delta t)}=69.3 \mathrm{~m} / \mathrm{s}^{2}$. Introducing the standard normal random variables $u_{i}=\ddot{b_{i}} / \sigma$, the above discrete representation of the white noise process is found to be of the form (1) with

$$
s_{i}(t)= \begin{cases}\sigma, & t_{i-1}<t \leq t_{i} \\ 0, & \text { otherwise }\end{cases}
$$

Note that this representation approximates the white noise by a band-limited broadband process with the upper frequency cutoff at approximately $1 /(2 \Delta t) \mathrm{Hz}$.

Assume the fragility of the product in the package is $20 \mathrm{~g}$, that is, $G_{c}=20 \mathrm{~g}$. The equivalent correction idealized linear system in this case can be

$$
m \ddot{z}+c_{\mathrm{eq}} \dot{z}+k_{\mathrm{eq}} z=m \ddot{b},
$$

where the free parameters are $\boldsymbol{\theta}=\left[k_{\mathrm{eq}}, c_{\mathrm{eq}}\right]$. One advantage of using this equivalent idealized linear system is that it is possible to construct correspondence between the properties of the original nonlinear system and the correction idealized linear system. For the cubic nonlinear packaging system in (24), we can expect that $c_{\text {eq }} \approx c$ and $k_{\text {eq }}>k$. Therefore, only one parameter $k_{\text {eq }}$ needs to be optimized. Let the start point of $k_{\text {eq }}=k$; in each iteration, the value of $k$ increases, and the reliability index $\beta_{i}$ is obtained by MCFM. In the iterations, when the minimum value of $\beta_{i}$ is obtained, the corresponding $k$ is $k_{\text {eq }}$. In this example, the optimization process of $k$ is shown in Figure 4.

From Figure 4, the optimized $k_{\text {eq }}$ in this example is $k_{\text {eq }}=955700 \mathrm{~N} / \mathrm{m}$, the corresponding reliability index $\beta=2.6599$, and the correction factor $v^{*}=1.0643$.

Obtaining the correction idealized linear system, in the standard normal space $u$, the hypersurface representing the limit-state function of the real nonlinear system can be replaced by the hyperplane representing the limit-state function of the correction idealized linear system at the design point (Figure 3 ). Therefore, the limit-state function of the system can be obtained by (21), and the FORM can be used to analyze the acceleration response reliability of the package. The reliability index is obtained by (13), the vector a $(t)$ is obtained by (10), $h(t)$ is obtained by (8), the vector $\mathbf{s}(\mathrm{t})$ can be obtained by (26), and the acceleration response first passage probability of the package is obtained by (4). In this example, the first passage failure probability of the package is calculated and shown in Figure 5. From Figure 5, the first passage failure probability of the package increases

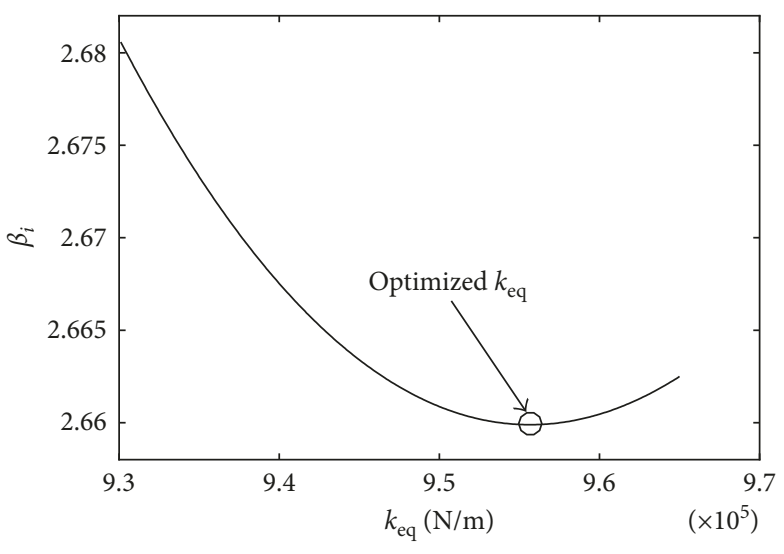

FIgURE 4: Optimization process of $k_{\text {eq }}$.

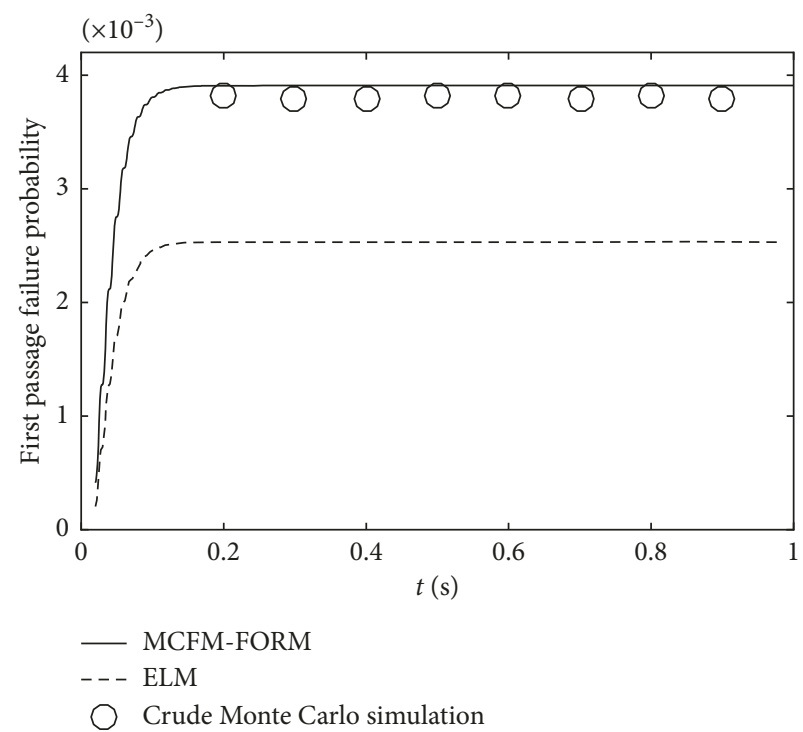

FIgURE 5: Acceleration response first passage failure probability of the nonlinear package.

approximately in an exponential way with time to a steady state, and the convergence time is less than $0.2 \mathrm{~s}$. To verify the effectiveness and accuracy of the method proposed in this paper, the crude Monte Carlo simulation is carried out to analyze the acceleration response failure probability of the package for comparison. In the Monte Carlo simulation, the number of simulations $N=10^{6}$, and the simulation of the base acceleration random vibration is realized by the harmonic superposition method [21]. From the comparison between the Monte Carlo simulation result and the analysis result using the MCFM-FORM, we can conclude the nonlinear package reliability analysis method presented in this paper has a good accuracy. The failure probability analysis result obtained by using the ELM method is also shown in Figure 5. The error of the analysis by the ELM is that the response of the nonlinear package excited by Gaussian excitation is non-Gaussian, while the model adopted in ELM can only estimate the second moments of the package response accurately in a general manner and cannot reproduce 


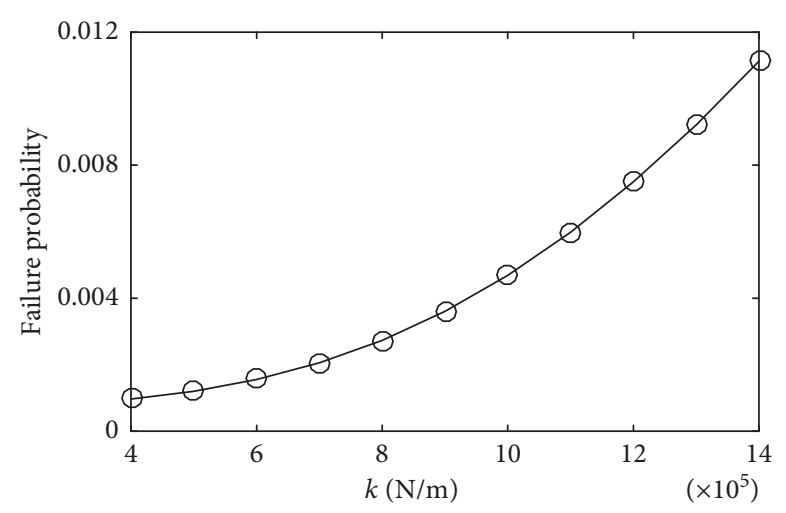

(a)

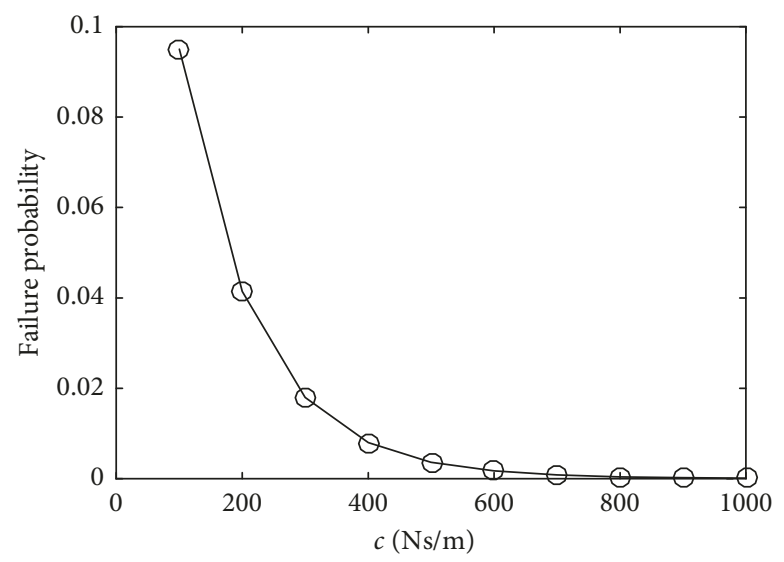

(c)

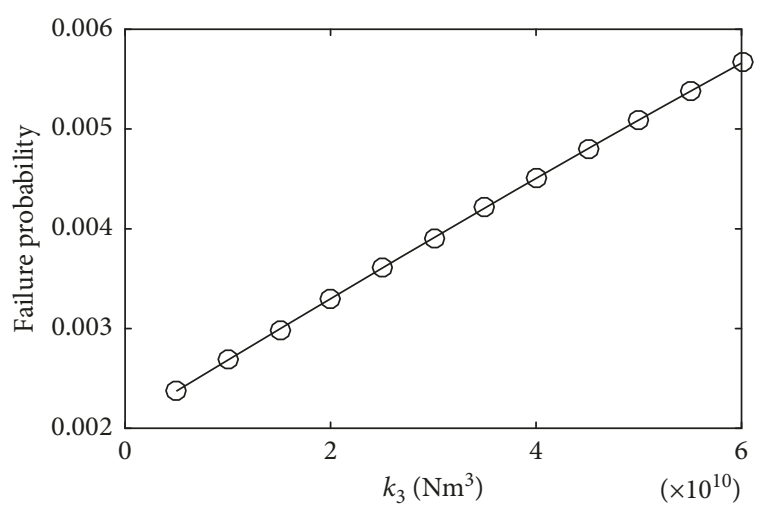

(b)

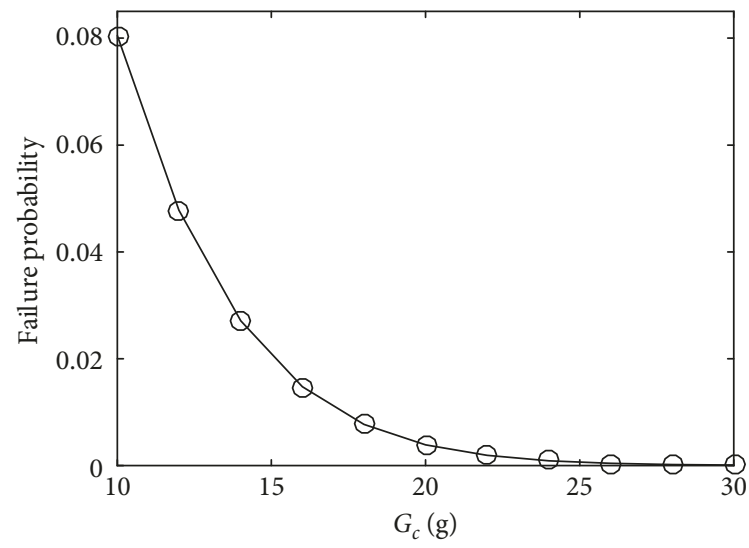

(d)

Figure 6: Effects of parameters variations on system failure probability.

higher moments of the response. The error of ELM is determined by the extent of the nonlinearity of the package and the value of threshold.

In order to find the effects of system parameters on package reliability, numerical simulations were carried out to observe the effects of parameters variations on the first passage failure probability. The parameters variation ranges were $k=4 \times 10^{5} \sim 14 \times 10^{5} \mathrm{~N} / \mathrm{m}, k_{3}=0.5 \times 10^{10} \sim 6 \times 10^{10} \mathrm{~N} / \mathrm{m}^{3}$, $c=100 \sim 1000 \mathrm{Ns} / \mathrm{m}$, and $G_{c}=10 \mathrm{~g} \sim 30 \mathrm{~g}$. The basic parameters are $c=491 \mathrm{Ns} / \mathrm{m}, k=930100 \mathrm{~N} / \mathrm{m}, k_{3}=3 \times 10^{10} \mathrm{~N} / \mathrm{m}^{3}$, and $G_{c}=20 \mathrm{~g}$. While one parameter varies in the set range, the other parameters remain constant as the basic parameters. We used the MCFM-FORM to analyze the failure probability of the system, and the effects of parameters variations on failure probability are shown in Figure 6.

From Figure 6, we can conclude the following:

(1) With the increase of the stiffness parameters $k$ and $k_{3}$, the first passage probability of the package also increases. This indicates that, for the package excited by white noise, a softer cushion in the package can lead to smaller package failure probability.

(2) Slight variation of damping can lead to a great change in failure probability, which means the failure probability is very sensitive to damping. We can conclude that the most effective method to improve vibration reliability of the package is to select a cushion material with good damping behavior in the package.

(3) The product fragility $G_{c}$ also affects the package vibration reliability, and higher product fragility can also improve the package reliability. Therefore, a durable product means good vibration reliability of the package.

\section{Conclusions}

In the package distribution process, most of the time, the package is base excited by random vibration, and it is necessary to analyze the package failure probability under random loads. The theory and method in the structure reliability field are implemented to analyze the failure probability of the nonlinear package.

The random loads are assumed to be white noise and are discretized in the standard normal space. A model correction factor method is employed to replace the real nonlinear model by an equivalent idealized model. Because of the simplicity and efficiency of the linear system, an equivalent linear package model is used to replace the nonlinear package model. An iterative algorithm is proposed to find the design point of the nonlinear system. At the design point, a hyperplane representing a linear system is used to replace the real hypersurface representing the nonlinear system. The FORM is used to obtain 
failure probability of the system. The accuracy and efficiency of the MCFM-FORM presented in this paper are verified by the crude Monte Carlo simulation. Numerical simulations are carried out to analyze the effects of each parameter on the failure probability. It can be concluded from simulations that the cushion material with softer stiffness and higher damping can improve vibration reliability of the package effectively, and the increase of product fragility can also lead to better vibration reliability behavior. The failure probability of the package is very sensitive to damping, and the most effective method to improve the vibration reliability behavior is the selection of cushion materials with higher damping property. The softer cushion material and higher product fragility can also improve the package vibration behavior.

In order to improve the accuracy of the package in the distribution process, the following topics are recommended for further research:

(1) Development of the package parameters sensitivity method. From parameters variation analysis (Figure 6), it is clear that the failure probability is sensitive to the variation of damping. Sensitivity measures are useful for many purposes, including identification of important sources of uncertainty and optimal package design.

(2) Development of the discrete representation for acceleration excitation with specified PSD characteristics. In order to improve the package failure probability analysis accuracy, consideration of the PSD characteristics of the acceleration excitation is inevitable. There can be many other approaches for representation of a continuous random process. For example, one can use the frequency domain analysis to represent a continuous random process. More investigation on the effective representation of a continuous random process is desirable.

\section{Data Availability}

The data used to support the findings of this study are available from the corresponding author upon request.

\section{Conflicts of Interest}

The author declares that there are no conflicts of interest regarding the publication of this paper.

\section{Acknowledgments}

This paper was supported by the National Natural Science Foundation of China (Grant no. 51765028) and the Innovation \& SYB Project of Lanzhou (Grant no. 2014A-049).

\section{References}

[1] Z. W. Wang and J. H. Jiang, "Evaluation of product dropping damage based on key component," Packaging Technology and Science, vol. 23, no. 4, pp. 227-238, 2010.

[2] J. H. Jiang and Z. W. Wang, "Dropping damage boundary curves for cubic and hyperbolic tangent packaging systems based on key component," Packaging Technology and Science, vol. 25, no. 7, pp. 397-411, 2012.

[3] C. Zhong, K. Saito, and K. Kawaguchi, "Improvement of equivalent drop theory for transport packaging," Packaging Technology and Science, vol. 26, no. 2, pp. 67-81, 2013.

[4] T. Piatkowski and P. Osowski, "Modified method for dynamic stress-strain curve determination of closed-cell foams," Packaging Technology and Science, vol. 29, no. 6, pp. 337-349, 2016.

[5] J. M. Gibert and G. S. Batt, "Impact oscillator model for the prediction of dynamic cushion curves of open cell foams," Packaging Technology and Science, vol. 28, no. 3, pp. 227-239, 2015.

[6] G. Li, V. Rouillard, and M. A. Sek, "Evaluation of static and dynamic cushioning properties of polyethylene foam for determining its cushion curves," Packaging Technology and Science, vol. 28, no. 1, pp. 47-57, 2015.

[7] F. D. Lu and D. Gao, "Quasi-static and impact responses of multi-layered corrugated paperboard cushion by virtual mass method," Packaging Technology and Science, vol. 27, no. 11, pp. 867-881, 2014.

[8] A. J. Chen, "The shock characteristics of tilted support spring packaging system with critical components," Shock and Vibration, vol. 2014, Article ID 496035, 8 pages, 2014.

[9] H. Li, A. Chen, and N. Duan, "Dropping shock characteristics of the suspension cushioning system with critical components," Shock and Vibration, vol. 2017, Article ID 3164294, 12 pages, 2017.

[10] M. Hao and A. J. Chen, "Dropping impact characteristics analysis of a cubic nonlinear packaging system with a cantilever beam type elastic critical component with concentrated tip mass," Shock and Vibration, vol. 2015, Article ID 602984, 10 pages, 2015.

[11] A. D. Kiureghian, "The geometry of random vibrations and solutions by FORM and SORM," Probabilistic Engineering Mechanics, vol. 15, no. 1, pp. 81-90, 2000.

[12] B. Keshtegar and M. Zeng, "A hybrid relaxed first-order reliability method for efficient structural reliability analysis," Structural Safety, vol. 66, pp. 84-93, 2017.

[13] H. Koo, A. D. Kiureghian, and K. Fujimura, "Design point excitation for nonlinear random vibrations," Probabilistic Engineering Mechanics, vol. 20, no. 2, pp. 136-147, 2005.

[14] K. Fujimura and A. D. Kiureghian, "Tail-equivalent linearization method for nonlinear random vibration," Probabilistic Engineering Mechanics, vol. 22, no. 1, pp. 63-76, 2007.

[15] A. D. Kiureghian and K. Fujimura, "Nonlinear stochastic dynamic analysis for performance-based earthquake engineering," Earthquake Engineering and Structural Dynamics, vol. 38, no. 5, pp. 719-738, 2009.

[16] A. Hadidi, B. F. Azar, and A. Rafiee, "Efficient response surface method for high-dimensional structural reliability analysis," Structural Safety, vol. 68, pp. 15-27, 2017.

[17] L. Faravelli, "Response-surface approach for reliability analysis," Journal of Engineering Mechanics, vol. 115, no. 12, pp. 2763-2781, 1989.

[18] A. I. Olsen and A. Naess, "An importance sampling procedure for estimating failure probabilities of non-linear dynamic systems subjected to random noise," International Journal of Non-Linear Mechanics, vol. 42, no. 6, pp. 848-863, 2007.

[19] T. T. Soong and M. Grigoriu, Random Vibration of Mechanical and Structural Systems, Prentice-Hall, Inc., Englewood Cliffs, NJ, USA, 1997.

[20] J. A. Nelder and R. Mead, "A simplex method for function minimization," Computer Journal, vol. 7, no. 4, pp. 308-313, 1965.

[21] A. Steinwolf, "Shaker random testing with low kurtosis: review of the methods and application for sigma limiting," Shock and Vibration, vol. 17, no. 3, pp. 219-231, 2010. 


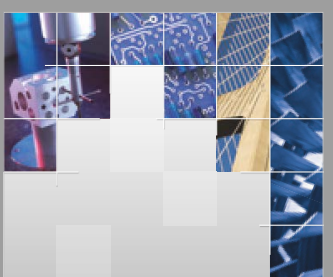

\section{Enfincering}
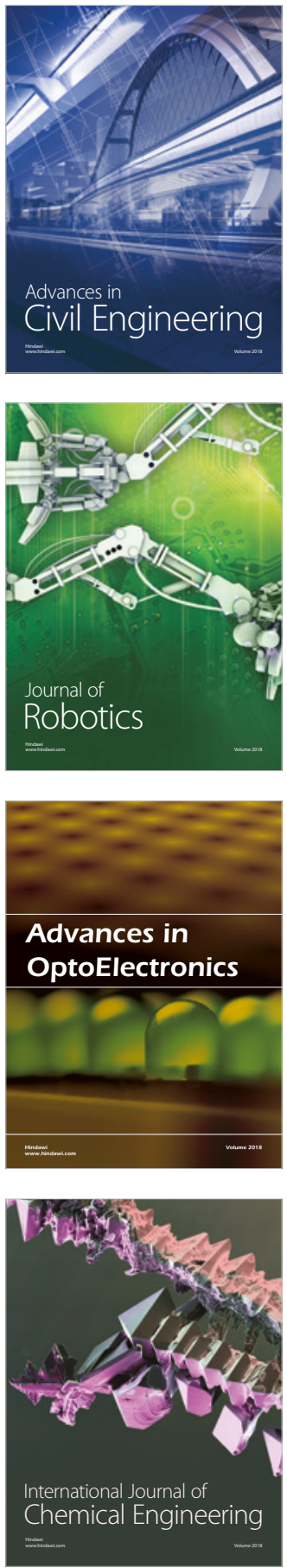

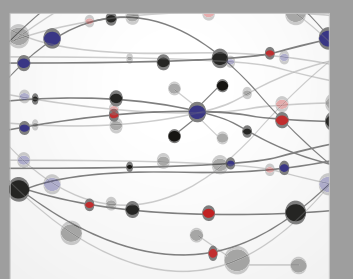

\section{Rotating \\ Machinery}

The Scientific World Journal

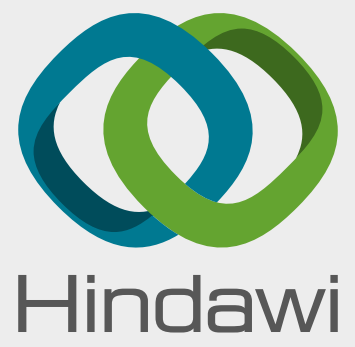

Submit your manuscripts at

www.hindawi.com
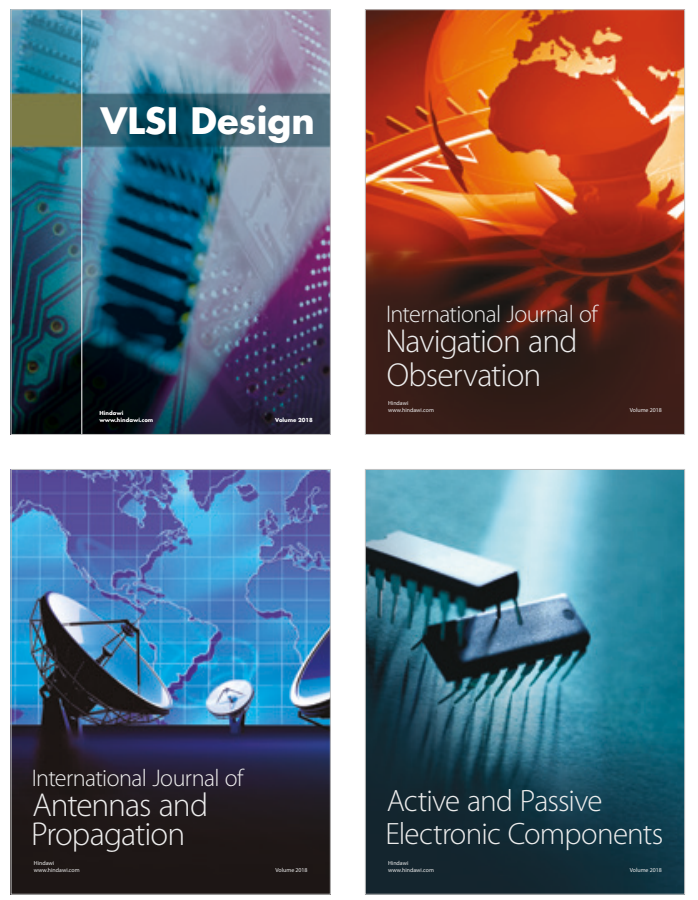
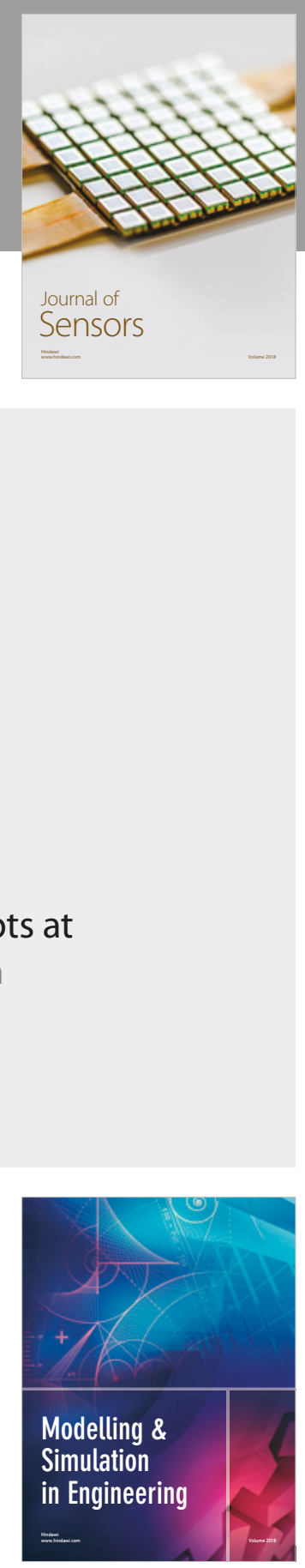

\section{Advances \\ Multimedia}
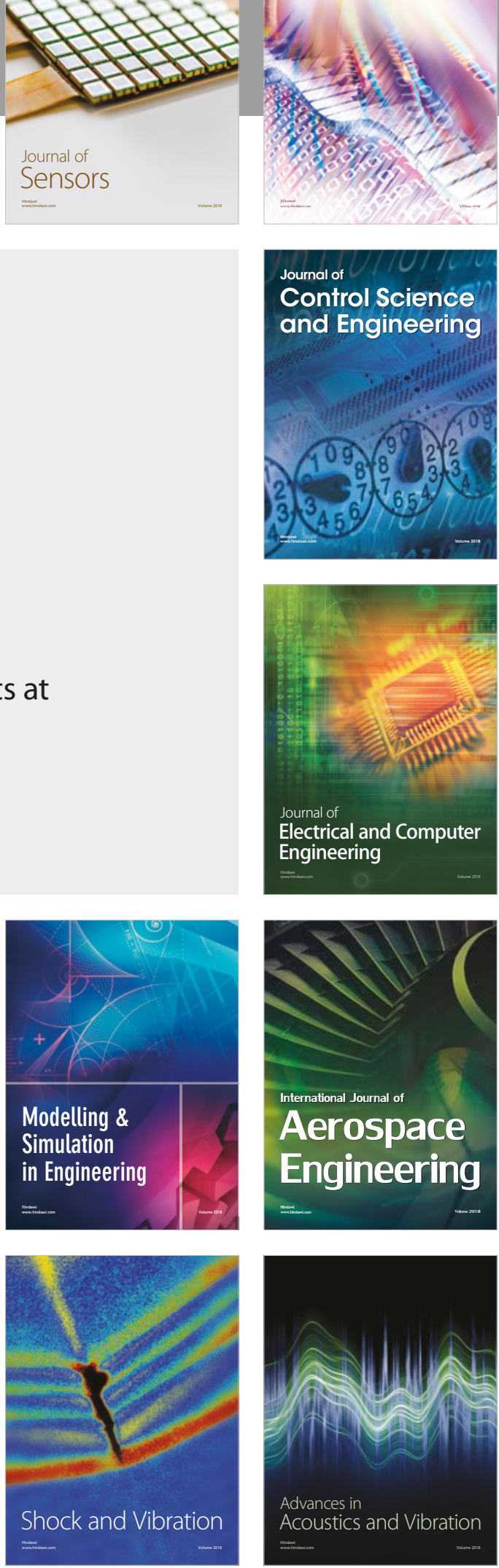